\title{
Wogonin suppresses osteopontin expression in adipocytes by activating PPAR
}

\author{
Ye-min ZHANG, Ming-xin LI, Zhao TANG, Chang-hua WANG* \\ Department of Pathophysiology, Wuhan University School of Basic Medical Sciences, Wuhan 430071, China
}

\begin{abstract}
Aim: Wogonin (5,7-dihydroxy-8-methoxyflavone), a major bioactive compound of the flavonoid family, is commonly extracted from the traditional Chinese medicine Scutellaria baicalensis and possesses antioxidant and anti-inflammatory activities and is assumed to have anti-diabetes function. Indeed, a current study has shown that it can possibly treat metabolic disorders such as those found in $d b / d b$ mice. However, the underlying molecular mechanism remains largely unclear. The aim of this study was to investigate the impact of wogonin on osteopontin (OPN) expression in adipose tissue from type 1 diabetic mice and in 3T3-L1 adipocytes.

Methods: Type 1 diabetes was induced by streptozotocin (STZ) injection. 3T3-L1 preadipocytes were converted to 3T3-L1 adipocytes through treatment with insulin, dexamethasone, and 3-isobutyl-1-methylxanthine (IBMX). Western blot analysis and RT-PCR were performed to detect protein expression and mRNA levels, respectively.

Results: Wogonin treatment suppressed the increase in serum OPN levels and reduced OPN expression in adipose tissue from STZinduced type 1 diabetic mice. Administration of wogonin enhanced PPAR $\alpha$ expression and activity. Silencing of PPAR $\alpha$ diminished the inhibitory effects of wogonin on OPN expression in 3T3-L1 adipocytes. Furthermore, the levels of c-Fos and phosphorylated c-Jun were reduced in wogonin-treated adipose tissue and 3T3-L1 adipocytes. In addition, wogonin treatment dramatically mitigated p38 MAPK phosphorylation. Pharmacological inhibition of p38 MAPK by its specific inhibitor SB203580 increased PPAR $\alpha$ activity and decreased OPN expression.

Conclusion: Our results suggest that wogonin downregulated OPN expression in adipocytes through the inhibition of p38 MAPK and the sequential activation of the PPAR $\alpha$ pathway. Given the adverse effects of high OPN levels on metabolism, our results provide evidence for the potential administration of wogonin as a treatment for diabetes.
\end{abstract}

Keywords: wogonin; osteopontin; PPAR $\alpha$; p38 MAPK; adipocytes

Acta Pharmacologica Sinica (2015) 36: 987-997; doi: 10.1038/aps.2015.37; published online 15 June 2015

\section{Introduction}

Osteopontin (OPN) is a multifunctional protein involved in several physiological and pathological events that serves as a biomarker or therapeutic target for such conditions as cancer, cardiovascular diseases, and various intractable inflammatory diseases ${ }^{[1-4]}$. Currently, OPN has been implicated in the pathogenesis of insulin resistance, obesity, and diabetes ${ }^{[5]}$. The pharmacological and genetic inhibition of OPN has mitigated hepatic and adipose tissue inflammation, improved obesityinduced insulin resistance in hepatic and adipose tissue, attenuated hepatic steatosis, and prevented the upregulation of gluconeogenic genes in the liver ${ }^{[6-9]}$, suggesting that OPN is an important player in the development of insulin resistance and diabetes.

Adipose tissue and adipocytes are two of the primary targets

\footnotetext{
* To whom correspondence should be addressed.

E-mail chwang0525@whu.edu.cn

Received 2015-01-14 Accepted 2015-03-30
}

for OPN action. A high level of OPN is associated with fat storage in adipose tissue, accumulation of adipose tissue macrophages, adipose tissue inflammation, and adipocyte hypertrophy ${ }^{[6-9]}$. In addition, adipocytes can synthesize OPN. In the early stage of high fat diet (HFD) consumption, adipose tissue showed altered OPN isoform expression, but total OPN protein was unchanged ${ }^{[8]}$. In agreement with this finding, high levels of OPN mRNA were found in the adipose tissues of obese and insulin-resistant humans and rats ${ }^{[8]}$. Mice exposed to chronic HFD exhibited dramatically increased plasma OPN levels and OPN protein abundance in adipose tissue ${ }^{[7,8]}$. Thus, inhibition of OPN production in adipocytes could be a novel approach for the treatment of insulin resistance and diabetes.

Wogonin (5,7-dihydroxy-8-methoxyflavone) is one of the major bioactive constituents of flavonoids from Scutellaria baicalensis, a traditional Chinese herbal medicine for the treatment of infection, inflammation, and cardiovascular diseases. Accumulating evidence suggests the therapeutic potential of wogonin for cancer treatment ${ }^{[10,11]}$. Current studies also 
identify wogonin as a functional modulator of endothelial cells $^{[12,13]}$. We have recently found that wogonin is a potent antioxidant capable of inhibiting lipotoxicity-induced apoptosis of vascular smooth muscle cells (VSMCs) via suppression of intracellular DAG generation ${ }^{[14]}$. Intriguingly, wogonin exhibits beneficial effects on glucose and lipid metabolism in $d b / d b$ mice $^{[15]}$, indicating that wogonin is a new candidate for the treatment of diabetes. However, the underlying molecular mechanism of wogonin action remains largely unknown.

Here, we report that wogonin treatment suppressed OPN expression in vivo and in vitro and blunted OPN secretion in 3T3-L1 adipocytes. We also demonstrated that the inhibitory effect of wogonin on OPN expression is largely due to its ability to activate PPARa. This novel finding adds further evidence in favor of wogonin as a potential diabetes treatment.

\section{Materials and methods}

\section{Antibodies and reagents}

The biochemical reagents and antibodies used in this study are as following: streptozotocin (STZ), fenofibric acid, and wogonin purchased from Sigma-Aldrich (St Louis, MO, USA); antibodies to OPN, PPARa, p38 MAPK, phospho-p38 MAPK, c-Fos, phospho-c-Jun, and histones purchased from Cell Signaling Technology (Beverly, MA, USA); anti-tubulin antibody, PPARa/shRNA lentiviral particles, and control shRNA lentiviral particles purchased from Santa Cruz Biotechnology (Santa Cruz, CA, USA); and secondary antibodies conjugated to alkaline phosphatase or horseradish peroxidase purchased from Promega (Madison, WI, USA).

\section{Animals and treatment}

C57BL/ 6 mice[provided by the Animal Biosafety Level 3 Laboratory (ABSL-3) of Wuhan University] at eight weeks of age were randomized into three groups: a control group, STZ-induced type 1 diabetes group, and wogonin treatment group. Type 1 diabetes was induced by a daily intraperitoneal injection (ip) of $55 \mathrm{mg} / \mathrm{kg}$ body weight STZ (dissolved in 0.1 mmol/L citric acid buffer, $\mathrm{pH} 4.5$ ) for 5 consecutive days ${ }^{[16,17]}$. After one week of STZ injection, mice were intraperitoneally administered either $10 \mathrm{mg} / \mathrm{kg}$ body weight of wogonin or vehicle control (20\% dimethyl sulfoxide and $80 \%$ saline) once every three days for 8 weeks. Because the $\mathrm{LD}_{50}$ of wogonin administered by intravenous injection in Sprague-Dawley rats was $286.15 \mathrm{mg} / \mathrm{kg}^{[18]}$, the doses or dose ranges used in this study were considered safe. At the end of wogonin treatment, the mice were euthanized, and adipose tissue and blood samples were harvested for experimentation. Mice were maintained at $23 \pm 2{ }^{\circ} \mathrm{C}$ and at $45 \% \pm 5 \%$ humidity with a $12: 12$ h lightdark cycle. The experiments were conducted according to the protocols approved by the Animal Care and Use Committee of Wuhan University.

\section{T3-L1 cell culture and differentiation}

3T3-L1 cells (ATCC, CL-173) were cultured in complete medium (DMEM supplemented with $10 \%$ FBS and $1 \%$ penicillin/streptomycin) at $37^{\circ} \mathrm{C}$ in a humidified atmosphere con- taining $5 \% \mathrm{CO}_{2}$. To convert 3T3-L1 fibroblasts to adipocytes, the cells were maintained in complete medium until they reached $100 \%$ confluence. Three days following confluence, the cells were incubated in complete medium supplemented with $1 \mu \mathrm{g} / \mathrm{mL}$ insulin, $0.25 \mu \mathrm{mol} / \mathrm{L}$ dexamethasone, and 0.5 $\mathrm{mmol} / \mathrm{L} 3$-isobutyl-1-methylxanthine (IBMX) for $3 \mathrm{~d}$, then transferred to complete medium containing $1 \mu \mathrm{g} / \mathrm{mL}$ insulin for another $3 \mathrm{~d}$, and finally maintained in complete medium for an additional $4 \mathrm{~d}$. At that time, approximately $90 \%$ of cells were considered to be mature adipocytes as confirmed by Oil Red O staining ${ }^{[19]}$.

\section{RT-PCR}

RT-PCR was performed as described previously ${ }^{[19]}$. Briefly, total RNA was isolated from mouse adipose tissues or 3T3L1 adipocytes using TRIzol reagent (Invitrogen, Carlsbad, CA, USA). The primers used in this study include mouse OPN: forward 5'-AGCCACAAGTTTCACAGCCACAAGG-3', reverse 5'-CTGAGAAATGAGCAGTTAGTATTCCTGC-3'; mouse PPARa: forward 5'-TCAGGGTACCACTACGGAGT-3', reverse 5'-CTTGGCATTCTTCCAAAGCG-3'; mouse acylCoA-oxidase (ACOX): forward 5'-ACTATATTTGGCCAATTTTGTG-3', reverse 5'-TGTGGCAGTGGTTTCCAAGCC-3'; mouse $\beta$-actin: forward 5'-CGGTTCCGATGCCCTGAGGCTCTT-3', reverse 5'-CGTCACACTTCATGATGGAATTGA-3'. PCR was performed in a Perkin-Elmer 9600 thermocycler. The cycling parameters were as follows: $50^{\circ} \mathrm{C}$ for $10 \mathrm{~min}$ and $95^{\circ} \mathrm{C}$ for $2 \mathrm{~min}$, followed by 26 cycles of a three-step cycle $\left(95^{\circ} \mathrm{C}\right.$ for $15 \mathrm{~s}, 60^{\circ} \mathrm{C}$ for $30 \mathrm{~s}$, and $72^{\circ} \mathrm{C}$ for $\left.2 \mathrm{~min}\right)$. PCR products were separated by electrophoresis through $1 \%$ agarose, and ethidium bromide stained bands were detected using a UV transilluminator.

\section{Nuclei isolation, Western blot and Pro-Q Diamond phospho- protein analysis}

Nuclei were isolated by commercially available nuclear and cytoplasmic extraction reagents (Bipec Biopharma Corporation, Cambridge, MA, USA) according to the manufacturer's instructions.

Total protein was extracted with lysis buffer containing $50 \mathrm{mmol} / \mathrm{L}$ HEPES (pH 7.5), $150 \mathrm{mmol} / \mathrm{L} \mathrm{NaCl}, 20 \mathrm{mmol} / \mathrm{L}$ sodium pyrophosphate, $10 \mathrm{mmol} / \mathrm{L} \mathrm{NaF}, 1 \mathrm{mmol} / \mathrm{L} \mathrm{NaVO}_{3}$, $2 \mathrm{mmol} / \mathrm{L}$ EDTA, 10\% glycerol, $1 \mathrm{mmol} / \mathrm{L} \mathrm{MgCl}, 1 \mathrm{mmol} / \mathrm{L}$ $\mathrm{CaCl}_{2}, 10 \%$ Nonidet-P40, $2 \mathrm{mmol} / \mathrm{L}$ PMSF, and 10\% phosphorylase inhibitor cocktail-I, -II, and -III. The protein concentration was determined using the BCA (bicinchoninic acid) protein assay reagent (Thermo, Rockford, IL, USA). For Western blot analysis, the samples in SDS-PAGE loading buffer were heated at $100^{\circ} \mathrm{C}$ for $5 \mathrm{~min}$. The proteins were separated by SDS-PAGE gel, transferred onto nitrocellulose membranes, and detected with specific antibodies ${ }^{[20]}$.

Phosphorylation analysis for PPARa was performed using the Pro-Q Diamond phosphoprotein gel stain according to the manufacturer's protocol (Molecular Probes, Invitrogen, Carlsbad, CA, USA). In brief, PPARa was immunoprecipitated and separated by SDS-PAGE gel. The levels of PPARa were deter- 
mined by Western blot analysis using a specific anti-PPARa antibody. Phosphoprotein bands were scanned by fluorescence at $532 \mathrm{~nm}^{[21]}$.

\section{Biochemical analysis}

OPN levels in the serum and culture medium were determined by an osteopontin mouse ELISA Kit (Abcam, Cambridge, MA, USA) according to the manufacturer's instructions. Serum insulin was measured using the rat/mouse insulin ELISA EZRMI-13K from EMD Millipore (Billerica, MA, USA) according to the provided protocol.

\section{Statistical analysis}

Each experiment in vitro was repeated at least 3 times with similar results. The data are presented as the mean \pm SEM. Results were analyzed using a one-way ANOVA followed by the Tukey-Kramer post hoc test and independent samples $t$-test. The differences were considered statistically significant at $P<0.05$.

\section{Results}

Wogonin ameliorated hyperglycemia and reduced OPN expression in vivo

To examine the potential impact of wogonin on glucose homeostasis, we compared serum glucose and insulin levels between the STZ-diabetes group and the wogonin treatment group. We found that wogonin-treated mice showed markedly lower glucose levels (Figure 1A) and higher insulin concentrations (Figure 1B) than STZ-diabetic mice.

To observe the impact of wogonin treatment on OPN synthesis and secretion, we first checked the expression of OPN protein and mRNA in perinephric white adipose tissue from STZ-diabetic mice using Western blot analysis and RT-PCR, respectively. As shown in Figure 1C and 1D, the levels of OPN protein and mRNA showed a dramatic upregulation in adipose tissue from the diabetic mice. As expected, wogonin treatment greatly decreased the expression of OPN protein and mRNA (compared to the STZ group: $P<0.05$ and $P<0.01$, respectively). Using a mouse-specific OPN ELISA kit, we found that the diabetic mice had an approximately 4.6-fold increase in serum OPN concentration (Figure 1E), from 64.3 $\mathrm{ng} / \mathrm{mL}$ to $296.9 \mathrm{ng} / \mathrm{mL}(P<0.01)$, whereas wogonin treatment significantly decreased serum OPN levels to $143.1 \mathrm{ng} / \mathrm{mL}$ $(P<0.05$, compared with the STZ group). These findings indicate that wogonin treatment significantly suppressed adipose tissue expression and secretion of OPN in the STZ-diabetic mice.

Wogonin downregulated OPN expression and secretion in 3T3-L1 adipocytes

To further confirm the effects of wogonin on OPN expression and secretion, 3T3-L1 pre-adipocytes were converted to 3T3-
A

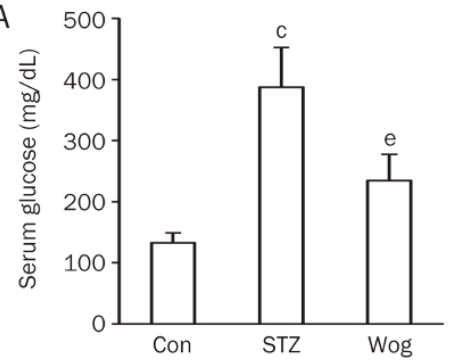

C
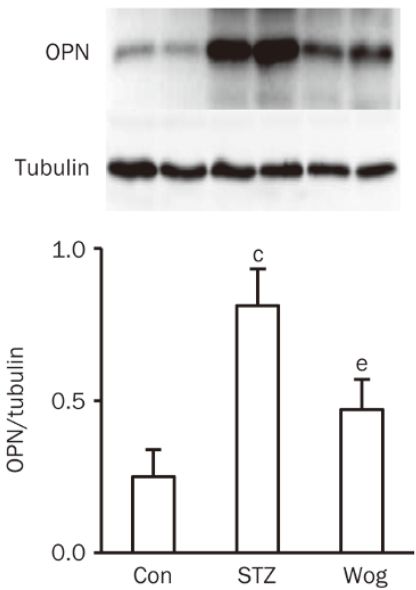

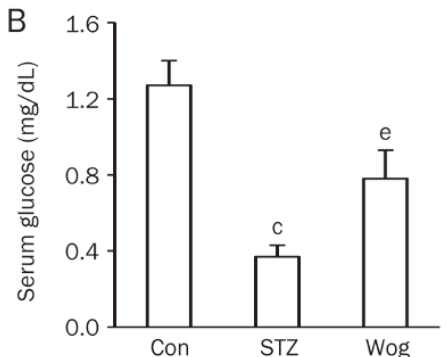

D
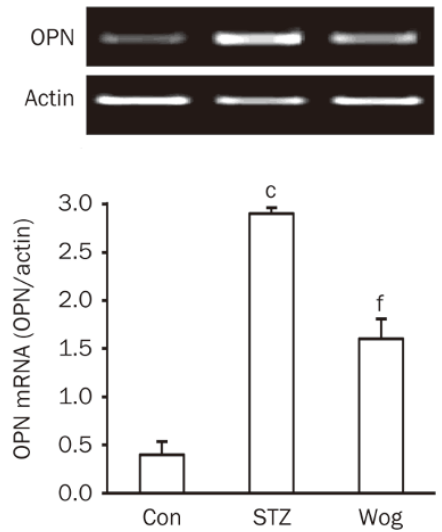

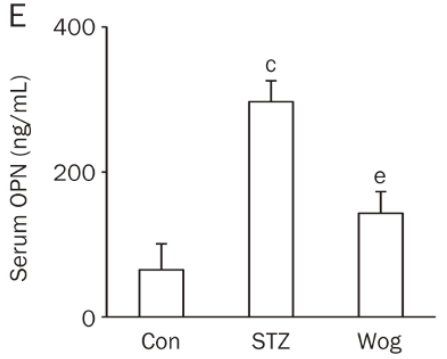

Figure 1. Wogonin improved hyperglycemia and suppressed OPN expression in STZ-induced type 1 diabetic mice. (A) Effect of wogonin on glucose levels. (B) Effect of wogonin on serum insulin concentration. (C and D) Effect of wogonin on the levels of OPN protein (C) and mRNA (D) in adipose tissue. (E) Effect of wogonin on serum OPN concentration. $n=12 .{ }^{c} P<0.01$ vs control group. ${ }^{e} P<0.05,{ }^{f} P<0.01$ vs STZ group. OPN, osteopontin; Con, control group; STZ, streptozotocin-induced type 1 diabetes group; Wog, wogonin treatment group. 
L1 adipocytes as previously described by our laboratory ${ }^{[19]}$. Then, 3T3-L1 adipocytes were incubated in complete medium containing $25 \mathrm{mmol} / \mathrm{L}$ glucose to mimic hyperglycemia. We found that high glucose stimulated the expression of OPN protein and mRNA, as well as OPN secretion (Supplementary Figure 1). When 3T3-L1 adipocytes were exposed to 25 $\mathrm{mmol} / \mathrm{L}$ glucose in the presence or absence of different concentrations of wogonin for desired time, we found that wogonin inhibited the expression of OPN protein in a doseand time-dependent manner (Figure 2A and 2B). To observe the impact of wogonin on OPN mRNA and secretion, 3T3-L1 adipocytes exposed to $25 \mathrm{mmol} / \mathrm{L}$ glucose were administered with $10 \mu \mathrm{mol} / \mathrm{L}$ wogonin for $12 \mathrm{~h}$. As depicted in Figure $2 \mathrm{C}$ and 2D, the levels of OPN mRNA in 3T3-L1 adipocytes (Figure 2C) and OPN protein in cultured medium (Figure 2D) were significantly decreased. These results are consistent with our in vivo findings, suggesting that wogonin inhibited OPN synthesis and secretion in 3T3-L1 adipocytes.

\section{Wogonin upregulated PPAR $\alpha$ expression and activity}

To investigate the underlying mechanism by which wogonin downregulated OPN production, we assessed the expression and activity of PPARa in wogonin-treated 3T3-L1 adipocytes and perinephric white adipose tissue from STZ-induced type 1 diabetic mice. 3T3-L1 adipocytes exposed to $25 \mathrm{mmol} / \mathrm{L}$ glucose were treated with $10 \mu \mathrm{mol} / \mathrm{L}$ wogonin for $12 \mathrm{~h}$. As shown in Figure 3A, wogonin greatly enhanced PPARa mRNA expression, but PPARa protein levels remained broadly unchanged (data not shown). Compared with STZ diabetic mice, wogonin treatment recovered STZ-reduced expression of PPARa mRNA (Figure 3B) and protein in both the cytosol (Figure 3C) and nucleus (Figure 3D) of adipose
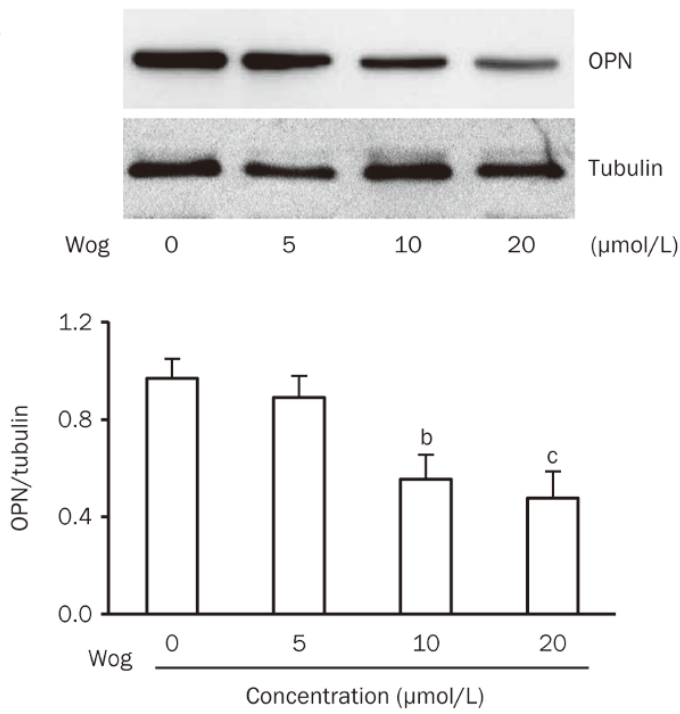

C
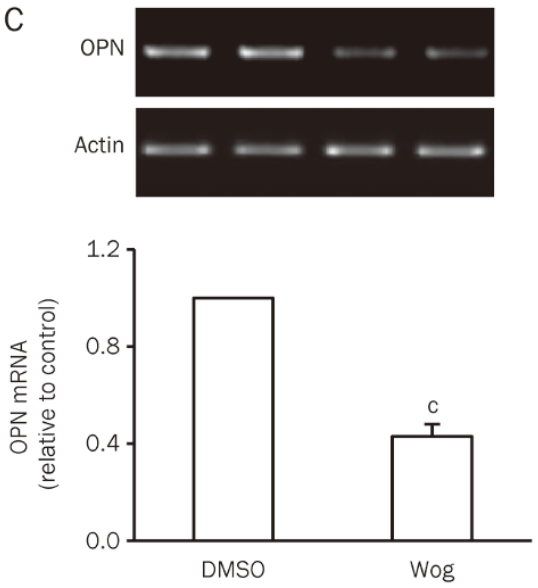

B
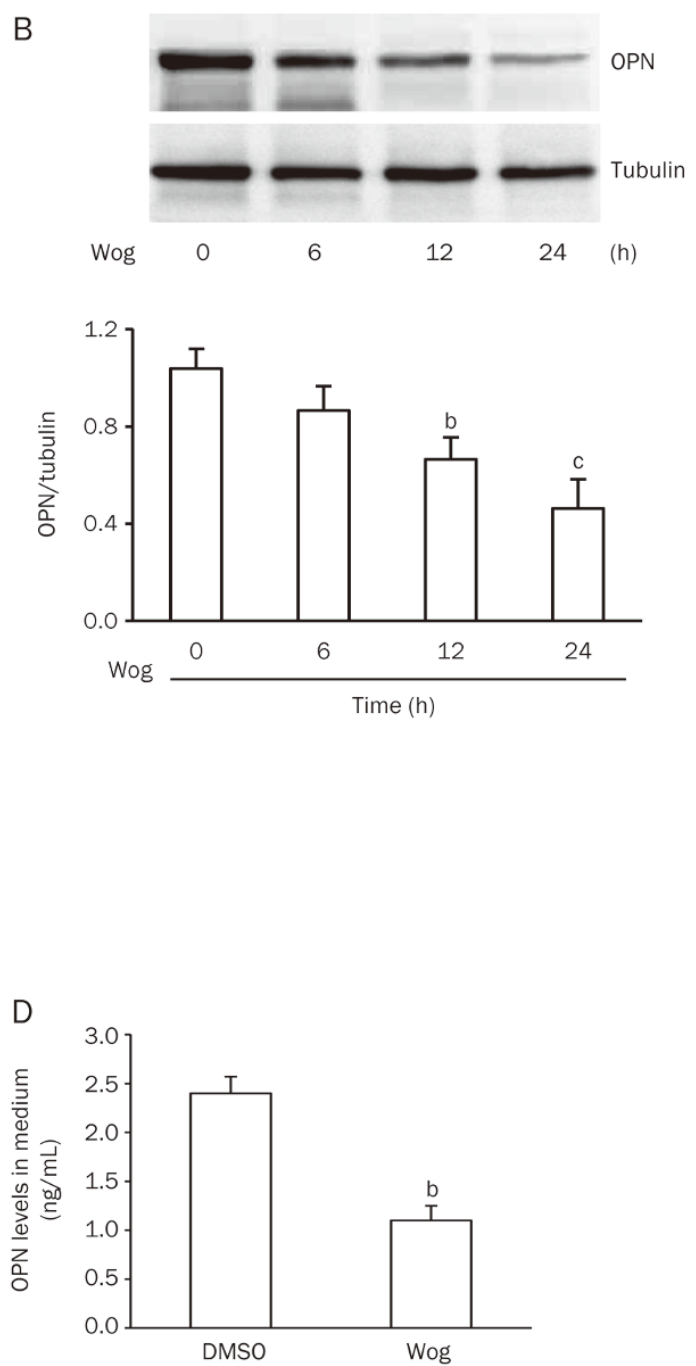

Figure 2. Wogonin inhibited OPN expression and secretion in 3T3-L1 adipocytes. (A and B) Dose-response (A) and time-response (B) of wogonin on OPN protein expression. 3T3-L1 adipocytes were incubated in complete medium containing $25 \mathrm{mmol} / \mathrm{L}$ glucose and received indicated concentrations of wogonin for $12 \mathrm{~h}(\mathrm{~A})$ or $10 \mu \mathrm{mol} / \mathrm{L}$ wogonin for the indicated time (B). (C) Effect of wogonin on the expression of OPN mRNA. (D) Effect of wogonin on OPN concentration in cultured medium. For ( $\mathrm{C}$ and D), 3T3-L1 adipocytes exposed to $25 \mathrm{mmol} / \mathrm{L}$ glucose were treated with or without $10 \mu \mathrm{mol} / \mathrm{L}$ wogonin for $12 \mathrm{~h} .{ }^{\mathrm{b}} \mathrm{P}<0.05,{ }^{\mathrm{c}} \mathrm{P}<0.01$ vs DMSO control group. OPN, osteopontin; Wog, wogonin. 
A
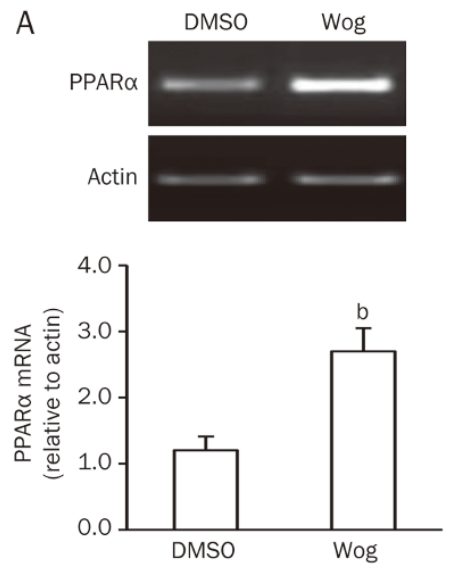

C
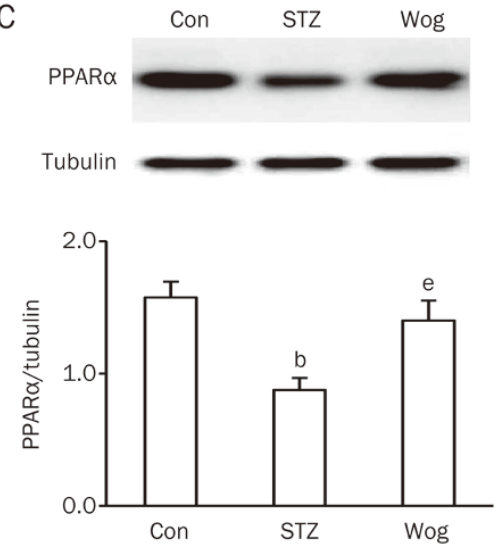

B
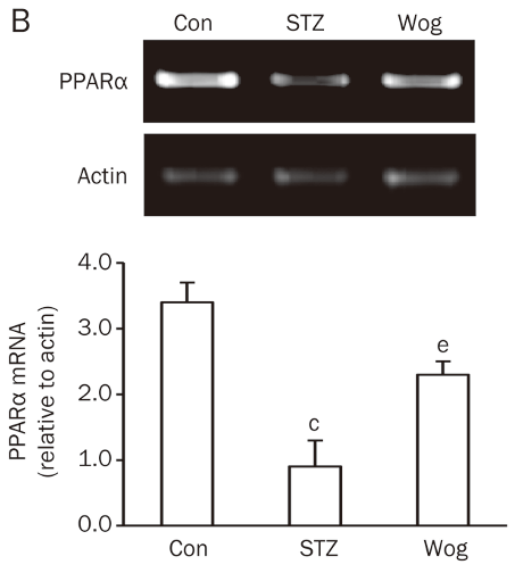

D
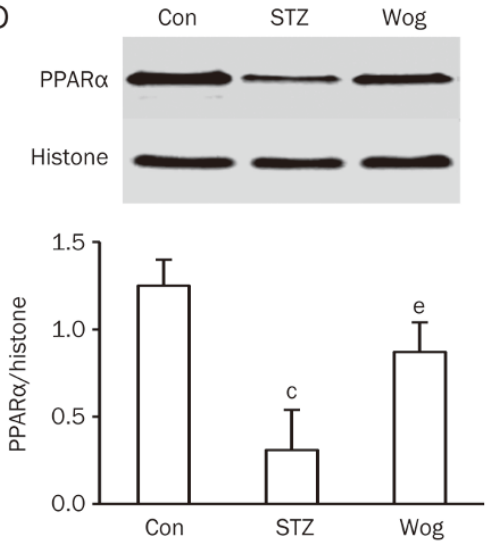

Figure 3. Wogonin increased PPAR $\alpha$ expression in adipose tissue and adipocytes. (A) Effect of wogonin on the mRNA expression of PPAR $\alpha$ in adipocytes. 3T3-L1 adipocytes exposed to $25 \mathrm{mmol} / \mathrm{L}$ glucose were treated with or without $10 \mu \mathrm{mol} / \mathrm{L}$ wogonin for $12 \mathrm{~h}$. (B and C) Effect of wogonin on PPAR $\alpha$ mRNA (B) and protein in the cytosol (C) in adipose tissues from STZ-diabetic mice $(n=12)$. (D) Effect of wogonin on PPAR $\alpha$ protein in the nucleus of adipose tissue from STZ-diabetic mice $(n=6)$. ${ }^{b} P<0.05,{ }^{c} P<0.01$ vs DMSO or control group. ${ }^{\mathrm{e}} P<0.05$ vs STZ-induced diabetic group. Con, control group; STZ, streptozotocin-induced type 1 diabetes group; Wog, wogonin treatment group.

tissue, suggesting that wogonin increased PPARa expression under high glucose conditions.

We next inspected the impact of wogonin on PPARa phosphorylation, which is closely associated with PPARa activity. 3T3-L1 adipocytes were cultured in complete medium containing $25 \mathrm{mmol} / \mathrm{L}$ glucose and treated with indicated concentrations of wogonin for $12 \mathrm{~h}$ (Figure 4A) or $10 \mu \mathrm{mol} / \mathrm{L}$ of wogonin for varied durations (Figure $4 \mathrm{~B}$ ). PPARa phosphorylation was determined by a Pro-Q Diamond phosphoprotein gel stain. We found that wogonin inhibited PPARa phosphorylation in a dose- and time-dependent manner in 3T3-L1 adipocytes (Figure 4A and 4B). Comparably, wogonin treatment inhibited STZ-stimulated PPARa phosphorylation (Figure 4C) in perinephric white adipose tissue from diabetic mice. Given that phosphorylation events can inhibit PPARa activity ${ }^{[21]}$, these data suggest that wogonin positively regulated PPARa activity in adipocytes. To support this conclusion, we measured PPARa transcriptional activity in a luciferase reporter gene assay. We found that wogonin significantly increased PPARa transactivation (Supplementary Figure 2). In agreement with this result, wogonin treatment reversed STZ-reduced mRNA expression of the PPARa target acyl-CoA oxidase (ACOX) in adipose tissue (Figure 4D).

Silencing of PPAR $\alpha$ diminished the inhibitory effect of wogonin on OPN expression in 3T3-L1 adipocytes

To further confirm the role of PPARa in mediating wogoninreduced OPN expression, 3T3-L1 adipocytes were infected with lentivirus carrying mouse PPARa/shRNA or scrambled sequences and then cultured in complete medium containing $25 \mathrm{mmol} / \mathrm{L}$ glucose followed by treatment with or without 10 $\mu \mathrm{mol} / \mathrm{L}$ wogonin for $12 \mathrm{~h}$. As expected, PPARa knockdown cells prevented the wogonin-induced reduction of OPN protein (Figure 5A) and mRNA (Figure 5B) and paralleled the restoration of OPN secretion (Figure 5C). In agreement with these results, the PPARa agonist fenofibrate significantly blocked the expression of OPN protein and mRNA, as well as OPN secretion in 3T3-L1 adipocytes (Supplementary Figure 3). However, a combined treatment with wogonin and fenofibrate did not further increase the impact of wogonin on OPN expression in 3T3-L1 adipocytes (Supplementary Figure 4).

It has been suggested that c-Fos and phospho-c-Jun play 

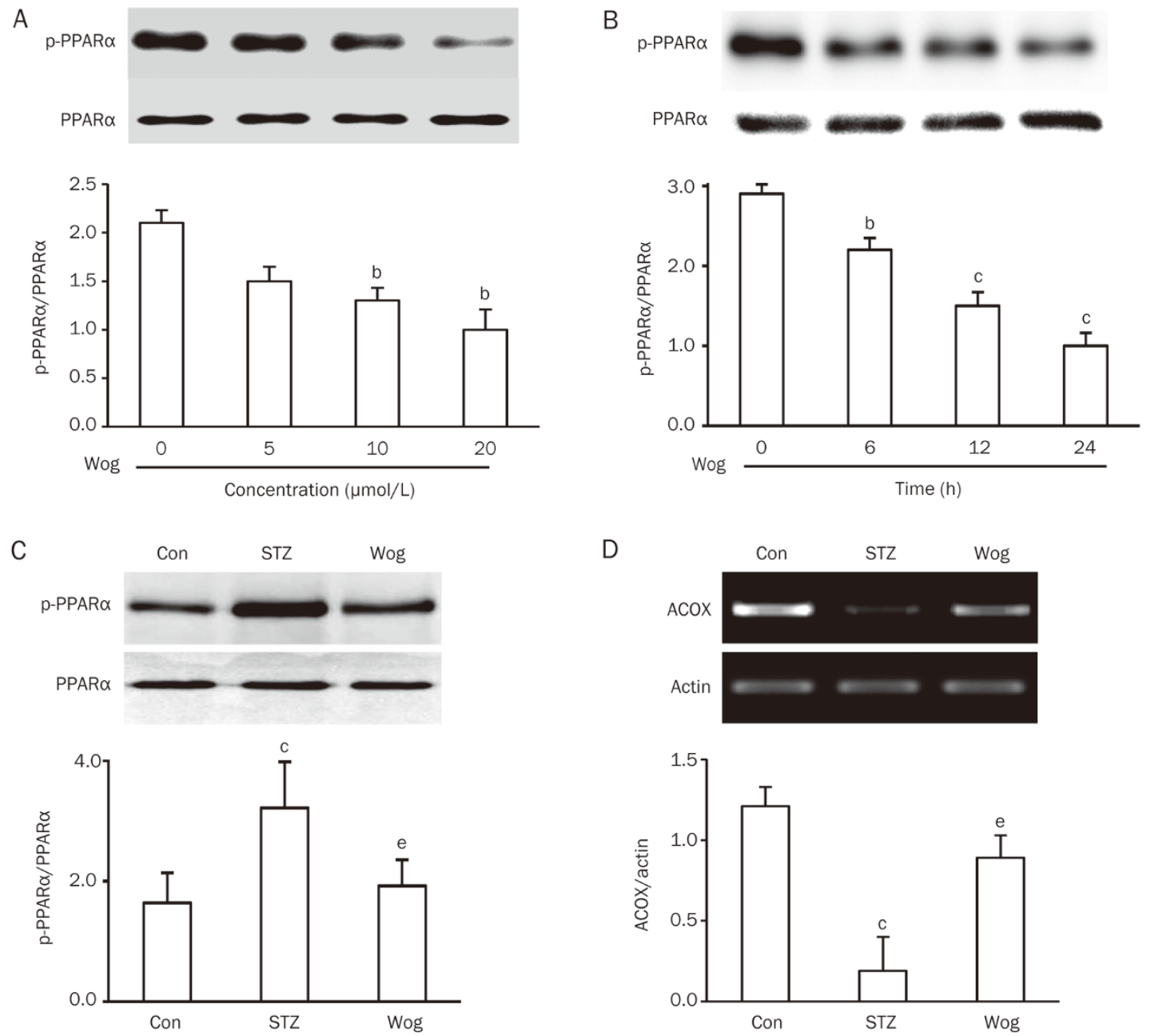

Figure 4. Wogonin inhibited PPAR $\alpha$ phosphorylation. (A and B) Dose- and time-response of wogonin on PPAR $\alpha$ phosphorylation in 3T3-L1 adipocytes. 3T3-L1 adipocytes were incubated in complete medium containing $25 \mathrm{mmol} / \mathrm{L}$ glucose and received indicated concentrations of wogonin for $12 \mathrm{~h}$ (A) or $10 \mu \mathrm{mol} / \mathrm{L}$ wogonin for the indicated time (B). (C) Effect of wogonin on PPAR $\alpha$ phosphorylation in adipose tissue from STZ-induced type 1 diabetic mice $(n=12)$. Cell extracts or tissues lysates were immunoprecipitated with anti-PPAR $\alpha$ antibody. The proteins were separated by SDS-PAGE and phosphoproteins were detected via Pro-Q Diamond phosphoprotein gel stain. (D) Effect of wogonin on the mRNA levels of the PPAR $\alpha$ target enzyme acyl-CoA oxidase (ACOX) in adipose tissue from STZ-induced type 1 diabetic mice $(n=6) .{ }^{b} P<0.05,{ }^{\mathrm{c}} P<0.01$ vs control group. ${ }^{\mathrm{e}} P<0.05$ vs STZ group. Con, control group; STZ, streptozotocin-induced type 1 diabetes group; Wog, wogonin treatment group.

a negative role in PPARa-reduced expression of OPN by acting on the proximal OPN promoter ${ }^{[22,23]}$. We next detected the expression of c-Fos and phospho-c-Jun in perinephric white adipose tissue and in 3T3-L1 adipocytes using Western blot analysis. As depicted in Figure 6A and 6B, wogonintreated adipose tissue exhibited lower expression of c-Fos and phospho-c-Jun in the nuclear fraction compared with the STZ group $(P<0.05, P<0.05$, respectively). Similar results were also found in 3T3-L1 adipocytes, demonstrating that wogonin treatment significantly repressed the expression of c-Fos (Figure 6C) and phospho-c-Jun (Figure 6D) in the nuclear fraction. Together, these results suggest PPARa mediated wogonin action on OPN expression.

\section{Wogonin inhibited p38 MAPK phosphorylation}

To investigate the potential role of p38 MAPK in wogonin- reduced OPN expression, we first studied the effect of wogonin on $\mathrm{p} 38$ phosphorylation in perinephric white adipose tissue from type 1 diabetic mice and 3T3-L1 adipocytes. As shown in Figure 7A, administration of wogonin inhibited STZ-increased phosphorylation of p38 MAPK in adipose tissue from diabetic mice $(P<0.05)$. When 3T3-L1 adipocytes were cultured in complete medium containing $25 \mathrm{mmol} / \mathrm{L}$ glucose and exposed to $10 \mu \mathrm{mol} / \mathrm{L}$ wogonin or $10 \mu \mathrm{mol} / \mathrm{L}$ of the specific p38 MAPK inhibitor SB203580 for $12 \mathrm{~h}$, p38 MAPK phosphorylation was significantly suppressed (Figure 7B). SB203580 was also found to inhibit PPARa phosphorylation (Figure 7C), which was accompanied by decreased OPN expression (Figure 7C and 7D). Additionally, the p38 MAPK inhibitor could not further enhance the inhibitory effects of wogonin on OPN expression in 3T3-L1 adipocytes (Supplementary Figure 5). These results suggest that p38 
A

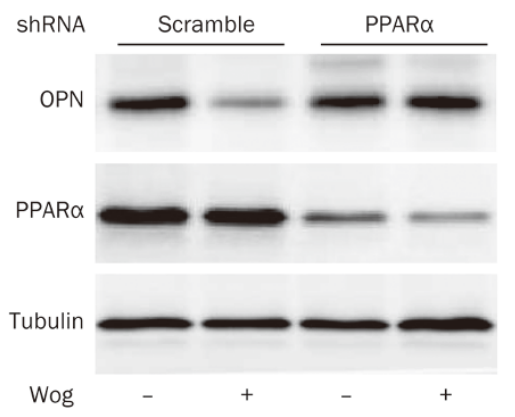

B

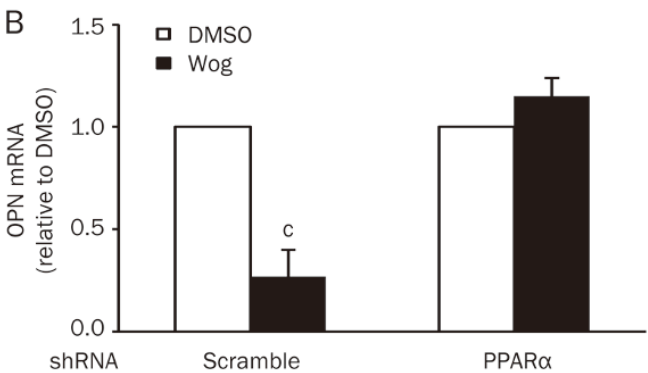

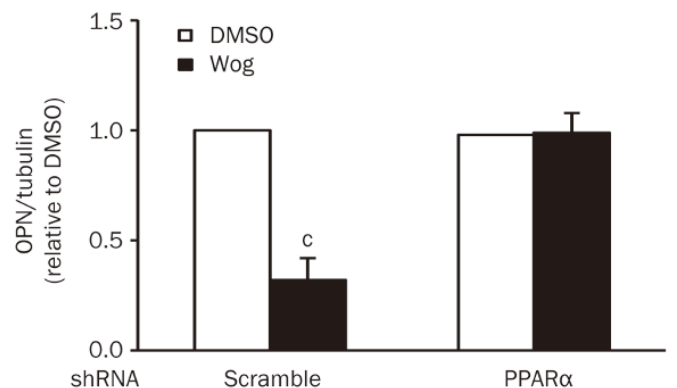

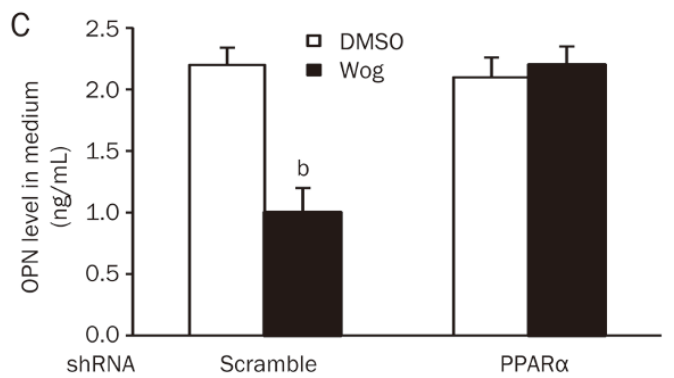

Figure 5. Silencing of PPAR $\alpha$ mitigated wogonin action on OPN synthesis and secretion in 3T3-L1 adipocytes. (A and B) Effect of PPAR $\alpha$ knockdown on the level of OPN protein (A) and mRNA (B) in 3T3-L1 adipocytes. (C) Effect of PPAR $\alpha$ knockdown on OPN concentration in culture medium. 3T3-L1 adipocytes infected with lentivirus carrying PPAR $\alpha /$ shRNA and scrambled sequences were cultured in complete medium containing $25 \mathrm{mmol} / \mathrm{L}$ glucose and followed by treatment with or without $10 \mu \mathrm{mol} / \mathrm{L}$ wogonin for $12 \mathrm{~h} .{ }^{b} P<0.05,{ }^{\mathrm{c}} P<0.01$ vs DMSO control group. OPN, osteopontin; Wog, wogonin.

MAPK is involved in the regulatory mechanism of wogonin for OPN expression.

\section{Discussion}

Oxidative stress and inflammation play a pivotal role in the pathogenesis of insulin resistance and diabetes ${ }^{[24,25]}$. Wogonin possesses anti-oxidant and anti-inflammatory properties ${ }^{[26,27]}$ and is assumed to have anti-diabetic activity. Recent studies have indeed shown that wogonin treatment in $d b / d b$ mice prevented weight gain, improved glucose tolerance, decreased serum insulin and cholesterol levels, and suppressed the accumulation of lipid droplets in the liver ${ }^{[15]}$. These pro-metabolic effects are associated with the enhanced expression of PPARa and adiponectin ${ }^{[15]}$. In this study, we demonstrate an inhibitory effect of wogonin on OPN expression in adipocytes in vivo and in vitro (Figure 1 and 2). Given that OPN is responsible for the severe inflammation and insulin resistance in insulin target tissue ${ }^{[6-9]}$, our results present a novel explanation for the anti-diabetic properties of wogonin.

Adipose tissue and adipocytes are two of the primary sites of OPN synthesis ${ }^{[5,28]}$. Previous studies have shown that OPN expression is strongly upregulated in adipose tissue from HFD-fed mice ${ }^{[7,9]}$. Our results found that STZ-induced type 1 diabetic mice exhibited higher OPN expression in adipose tissue (Figure 1), suggesting that the upregulation of OPN mRNA and protein exists similarly in the adipose tissue of type 1 diabetic mice, which may be due to high blood glucose or hyperglycemia ${ }^{[29,30]}$. In fact, high OPN expression and hyperglycemia could influence each other. STZ-induced hyperglycemia lead to the acute upregulation of serum OPN levels and pancreatic OPN mRNA and protein ${ }^{[29]}$. In cultured rat aortic smooth muscle cells, high glucose has been shown to stimulate OPN expression ${ }^{[30]}$. In contrast, OPN deficiency prevented glucose production and hyperglycemia in HFD mice $^{[6,9]}$. These studies suggest that the interplay between OPN and hyperglycemia may form a vicious cycle, leading to the development of diabetes. Wogonin could interfere with this cycle, thus exhibiting beneficial results for patients with diabetes.

PPARa plays a central role in the regulation of energy metabolism, particularly in lipid and lipoprotein metabolism. Recently, several studies have shown that PPARa is expressed in the adipose tissues of humans and rodents ${ }^{[31,32]}$. In both type 1 and type 2 diabetes models, PPARa expression was significantly downregulated in some tissues ${ }^{[15,33]}$, consistent with our results showing that expression levels of PPARa protein and mRNA in adipose tissues of diabetic mice were statistically significantly decreased compared to those of control mice (Figure 3B-3D). Our results further confirm that wogonin treatment increased PPARa expression (Figure 3), inhibited PPARa phosphorylation (Figure 4A-4C), enhanced PPARa transactivation (Supplementary Figure 2), and restored mRNA expression of the PPARa target ACOX (Figure $4 \mathrm{D}$ ) in the adipose tissue of diabetic mice and/or in 3T3-L1 adipocytes. PPARa phosphorylation has been suggested to have a negative association with PPARa activity; decreased phosphorylation leads to increased PPARa activity ${ }^{[21]}$. Hence, our results indicate that wogonin could activate PPARa, in 
A
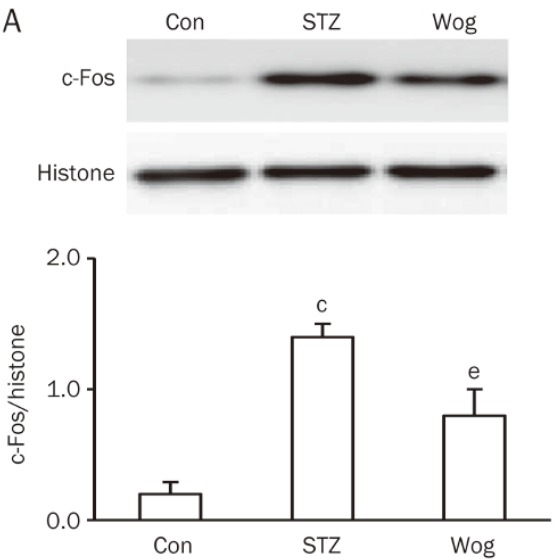

C
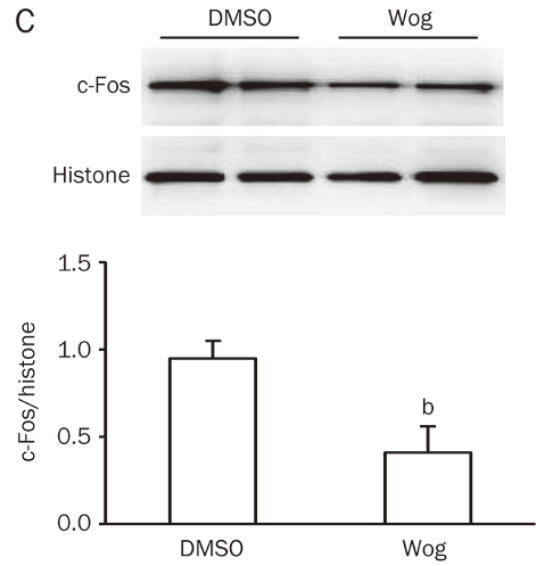

B
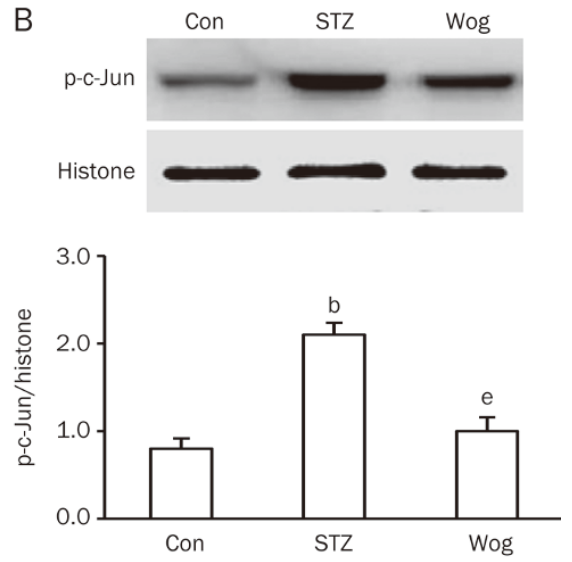

D
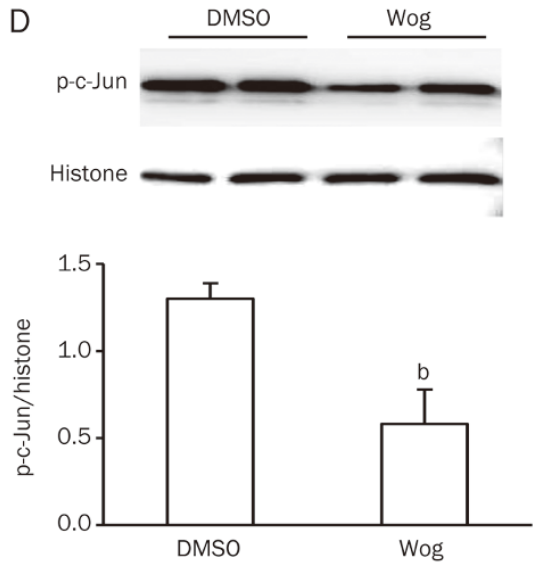

Figure 6. Wogonin inhibited the expressions of C-Fos and phospho-C-Jun in adipose tissue and 3T3-L1 adipocytes. (A and B) Effect of wogonin on the expression of c-Fos (A) and the phosphorylation status of C-Jun (B) in adipose tissue from STZ-induced type 1 diabetic mice ( $n=12$ ). (C and D) Effect of wogonin on the expression of c-Fos (C) and phosphorylation status of c-Jun (D) in 3T3-L1 adipocytes ( $n=4)$. 3T3-L1 adipocytes cultured in high-glucose complete medium were treated with or without $10 \mu \mathrm{mol} / \mathrm{L}$ wogonin for $12 \mathrm{~h} .{ }^{\mathrm{b}} P<0.05,{ }^{\mathrm{c}} P<0.01$ vs control or DMSO group. ${ }^{\mathrm{e}} P<0.05$ vs STZ group. Con, control group; STZ, streptozotocin-induced type 1 diabetes group; Wog, wogonin treatment group.

agreement with a previous study showing that wogonin stimulated PPARa expression and activity in the liver and white adipose tissue of $d b / d b$ mice ${ }^{[15]}$. Furthermore, our results showed that PPARa knockdown abrogated the inhibitory effects of wogonin on OPN expression in 3T3-L1 adipocytes (Figure 5), suggesting that PPARa mediated the OPN lowering effects of wogonin.

Accumulating evidence has suggested that c-Fos and c-Jun are involved in PPARa-induced reduction of OPN expression. A PPARa agonist downregulated the expression of c-Fos and phosphorylated c-Jun and inhibited its association with the OPN promoter, leading to reduced OPN expression. Overexpression of c-Fos and c-Jun abolished the inhibitory effect of PPARa on OPN expression. These results suggest that PPARa negatively regulates OPN expression through negative interference with c-Fos/c-Jun on the proximal OPN promoter $^{[22,23]}$. Our results indicated that wogonin inhibited the protein expression of c-Fos and phospho-c-Jun in adipose tissue from diabetic mice and in 3T3-L1 adipocytes (Figure 6). These findings, combined with our observation that wogonin upregulated PPARa expression and activity, further support the notion that PPARa mediates the inhibitory action of wogonin on OPN expression.

The remaining question is how PPARa is activated by wogonin. Previous studies have found convincing evidence that p38 MAPK is a kinase upstream of PPARa. Indeed, the impact of p38 MAPK on PPARa activity is dependent upon cell type and its physiological or pathophysiological condition. Activation of p38 MAPK in cardiomyocytes led to increased PPARa transcriptional activity ${ }^{[34]}$. Conversely, the p38 activator anisomycin induced a dose-dependent phosphorylation of PPARa and a $50 \%$ inhibition of its transcriptional activity in COS-7 cells ${ }^{[21]}$. Our results indicated that wogonin treatment dramatically mitigated p38 MAPK phosphorylation (Figure 7A and 7B), consistent with early studies showing that the expression of phosphorylated p38 MAPK was significantly attenuated by treatment with wogonin $^{[26,35]}$. In addition, the inhibition of p38 MAPK by its specific inhibitor SB203580 decreased PPARa phosphorylation (Figure 7C) and OPN expression (Figure 7C and 7D). These 
A
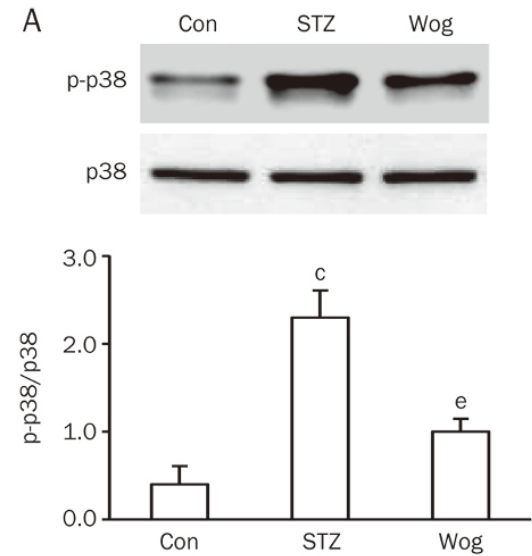

C

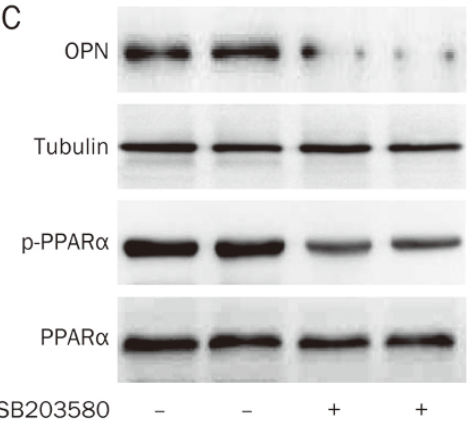

B
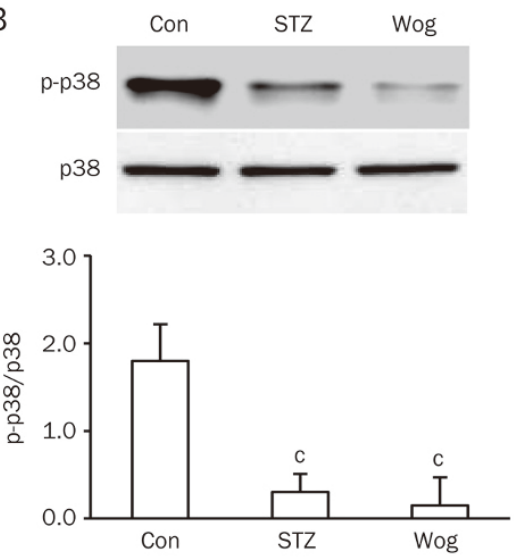

D

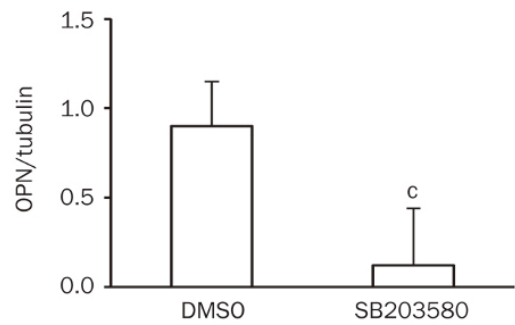

Figure 7. p38 MAPK mediated wogonin action on OPN expression. (A and B) Effect of wogonin on p38 MAPK phosphorylation in adipose tissue from STZ-induced type 1 diabetic mice (A, $n=12$ ) and 3T3-L1 adipocytes (B, $n=4)$. (C) Effect of the specific p38 MAPK inhibitor SB203580 on OPN expression and PPAR $\alpha$ phosphorylation. (D) Quantification of OPN levels in SB203580-treated adipocytes in (A). ${ }^{\mathrm{c}} P<0.01$ vs control or DMSO group. ${ }^{e} P<0.05$ vs STZ group. Con, control group; STZ, streptozotocin-induced type 1 diabetes group; Wog, wogonin treatment group.

findings suggest that p38 MAPK negatively impacts PPARa regulation activity in adipocytes. Interestingly, basal p38 MAPK phosphorylation was significantly elevated in type 2 diabetic adipocytes ${ }^{[36]}$. Activation of p38 MAPK has also been observed in response to such stress stimuli as STZ administration, inflammation, and high glucose ${ }^{[37-40]}$. Hence, increased p38 MAPK activation may be involved in the development of insulin resistance and diabetes. Our findings implicate p38 MAPK in the regulation of PPARa activity and sequential OPN expression ${ }^{[41]}$.

Traditionally, inflammatory cells are considered a major source of serum OPN in cases of morbid obesity ${ }^{[9,42]}$. The contributions of adipose tissue and adipocytes to the circulating levels of OPN remain largely unknown. A previous study has shown a highly significant positive correlation between plasma OPN levels and body fat percentage ${ }^{[43]}$. In contrast, You et al reported that serum OPN concentration was not associated with body fat percentage ${ }^{[44]}$. However, these two studies have demonstrated that fat loss produced a statistically significant reduction in circulating OPN concentration ${ }^{[43,44]}$. In addition, high levels of serum OPN were found in obese individuals $^{[42,43,45]}$. These findings suggest that adipocytes may be one origin of circulating OPN levels.

In the present study, we used 3T3-L1 adipocytes to probe the possibility of induced OPN secretion by adipocytes under high glucose and hyperglycemic conditions. Our results showed that high glucose stimulated OPN secretion in 3T3L1 adipocytes (Supplementary Figure 1), which was inhibited by wogonin treatment (Figure 2D), suggesting a potential involvement of adipocytes in the regulation of serum OPN concentration. Combined with our findings showing that wogonin attenuated an increase in STZ-induced serum OPN concentration (Figure 1E), our results indicated that adipocytes were at least partially responsible for the increased serum OPN concentration under STZ-induced hyperglycemic status. However, we still cannot conclude that adipocytes are the main source of serum OPN due to the multiple impacts of wogonin on factors related to metabolic disorders. For example, the anti-oxidative, anti-inflammatory, and antihyperglycemic activities of wogonin may be directly or indirectly involved in the regulation of OPN secretion by other cell types such as inflammatory cells. Further studies using adipose-specific OPN knockout mice are needed to determine the contribution of adipocytes to the serum OPN concentration. Additionally, we also do not know the detailed mechanism of secretion inhibition by wogonin. One possibility is that diminished OPN generation in adipocytes results in decreased secretion. 
Collectively, our results demonstrate that wogonin suppresses OPN expression through a mechanism involving p38 MAPK inhibition and sequential PPARa activation in adipocytes. Thus, targeting OPN by wogonin provides a novel therapeutic strategy for the treatment of insulin resistance and diabetes.

\section{Acknowledgements}

We thank Dr Leyuan Jack CHEN (Texas Tech University Health Sciences Center) for editing the manuscript for English grammar. The study was supported by a grant from the National Natural Science Foundation of China (No 81170790/ H0718) to Dr Chang-hua WANG.

\section{Author contribution}

Chang-hua WANG and Ye-min ZHANG designed the research; Ye-min ZHANG, Ming-xin LI, and Zhao TANG performed the research; Chang-hua WANG analyzed the data and wrote the paper.

\section{Supplementary information}

Supplementary figures are available at the Acta Pharmacologica Sinica's website.

\section{References}

1 Singh M, Dalal S, Singh K. Osteopontin: at the cross-roads of myocyte survival and myocardial function. Life Sci 2014; 118: 1-6.

2 Wolak T. Osteopontin - a multi-modal marker and mediator in atherosclerotic vascular disease. Atherosclerosis 2014; 236: 327-37.

3 Bandopadhyay M, Bulbule A, Butti R, Chakraborty G, Ghorpade P, Ghosh P, et al. Osteopontin as a therapeutic target for cancer. Expert Opin Ther Targets 2014; 18: 883-95.

4 Inoue M, Shinohara ML. Intracellular osteopontin (iOPN) and immunity. Immunol Res 2011; 49: 160-72.

5 Kahles F, Findeisen HM, Bruemmer D. Osteopontin: a novel regulator at the cross roads of inflammation, obesity and diabetes. Mol Metab 2014; 3: 384-93.

6 Kiefer FW, Neschen S, Pfau B, Legerer B, Neuhofer A, Kahle M, et al. Osteopontin deficiency protects against obesity-induced hepatic steatosis and attenuates glucose production in mice. Diabetologia 2011; 54: 2132-42.

7 Kiefer FW, Zeyda M, Gollinger K, Pfau B, Neuhofer A, Weichhart T, et al. Neutralization of osteopontin inhibits obesity-induced inflammation and insulin resistance. Diabetes 2010; 59: 935-46.

8 Chapman J, Miles PD, Ofrecio JM, Neels JG, Yu JG, Resnik JL, et al. Osteopontin is required for the early onset of high fat diet-induced insulin resistance in mice. PLoS One 2010; 5: e13959.

9 Nomiyama T, Perez-Tilve D, Ogawa D, Gizard F, Zhao Y, Heywood EB, et al. Osteopontin mediates obesity-induced adipose tissue macrophage infiltration and insulin resistance in mice. J Clin Invest 2007; 117: 2877-88.

10 Chirumbolo S. Anticancer properties of the flavone wogonin. Toxicology 2013; 314: 60-4.

11 Li-Weber M. New therapeutic aspects of flavones: the anticancer properties of Scutellaria and its main active constituents Wogonin, Baicalein and Baicalin. Cancer Treat Rev 2009; 35: 57-68.

12 Lee SO, Jeong YJ, Yu MH, Lee JW, Hwangbo MH, $\mathrm{Kim} \mathrm{CH}$, et al. Wogonin suppresses TNF-alpha-induced MMP-9 expression by blocking the NF-kappaB activation via MAPK signaling pathways in human aortic smooth muscle cells. Biochem Biophys Res Commun 2006; 351: 118-25.

13 Chang YL, Shen JJ, Wung BS, Cheng JJ, Wang DL. Chinese herbal remedy wogonin inhibits monocyte chemotactic protein-1 gene expression in human endothelial cells. Mol Pharmacol 2001; 60: 507-13.

14 Liu YM, Wang X, Nawaz A, Kong ZH, Hong Y, Wang CH, et al. Wogonin ameliorates lipotoxicity-induced apoptosis of cultured vascular smooth muscle cells via interfering with DAG-PKC pathway. Acta Pharmacol Sin 2011; 32: 1475-82.

15 Bak EJ, Kim J, Choi YH, Kim JH, Lee DE, Woo GH, et al. Wogonin ameliorates hyperglycemia and dyslipidemia via PPAR $\alpha$ activation in $d b / d b$ mice. Clin Nutr 2014; 33: 156-63.

16 Huynh K, Kiriazis H, Du XJ, Love JE, Gray SP, Jandeleit-Dahm KA, et al. Targeting the upregulation of reactive oxygen species subsequent to hyperglycemia prevents type 1 diabetic cardiomyopathy in mice. Free Radic Biol Med 2013; 60: 307-17.

17 Bai Y, Cui W, Xin Y, Miao X, Barati MT, Zhang C, et al. Prevention by sulforaphane of diabetic cardiomyopathy is associated with upregulation of Nrf2 expression and transcription activation. J Mol Cell Cardiol 2013; 57: 82-95.

18 Qi Q, Peng J, Liu W, You Q, Yang Y, Lu N, et al. Toxicological studies of wogonin in experimental animals. Phytother Res 2009; 23: 417-22.

19 Sun S, Liu Y, Lu J, Omar A, Sun S, Bi Y, et al. The inhibitory effects of PKC $\theta$ on adiponectin expression is mediated by ERK in 3T3-L1 adipocytes. J Endocrinol Invest 2011; 34: 8-15.

20 Wang X, Yu W, Nawaz A, Guan F, Sun S, Wang C. Palmitate induced insulin resistance by PKCtheta-dependent activation of mTOR/S6K pathway in C2C12 myotubes. Exp Clin Endocrinol Diabetes 2010; 118: 657-61.

21 Diradourian C, Le May C, Caüzac M, Girard J, Burnol AF, Pégorier JP. Involvement of ZIP/p62 in the regulation of PPARalpha transcriptional activity by p38-MAPK. Biochim Biophys Acta 2008; 1781: 239-44.

22 Nakamachi T, Nomiyama T, Gizard F, Heywood EB, Jones KL, Zhao Y, et al. PPARalpha agonists suppress osteopontin expression in macrophages and decrease plasma levels in patients with type 2 diabetes. Diabetes 2007; 56: 1662-70.

23 Li CB, Li XX, Chen YG, Zhang C, Zhang MX, Zhao XQ, et al. Effects and mechanisms of PPARalpha activator fenofibrate on myocardial remodelling in hypertension. J Cell Mol Med 2009; 13: 4444-52.

24 Rochette L, Zeller M, Cottin Y, Vergely C. Diabetes, oxidative stress and therapeutic strategies. Biochim Biophys Acta 2014; 1840: 2709-29.

25 Fernández-García JC, Cardona F, Tinahones FJ. Inflammation, oxidative stress and metabolic syndrome: dietary modulation. Curr Vasc Pharmacol 2013; 11: 906-19.

26 Lee YM, Cheng PY, Chen SY, Chung MT, Sheu JR. Wogonin suppresses arrhythmias, inflammatory responses, and apoptosis induced by myocardial ischemia/reperfusion in rats. J Cardiovasc Pharmacol 2011; 58: 133-42.

27 Huang $\mathrm{WH}$, Lee AR, Yang $\mathrm{CH}$. Antioxidative and anti-inflammatory activities of polyhydroxyflavonoids of Scutellaria baicalensis GEORGI. Biosci Biotechnol Biochem 2006; 70: 2371-80.

28 Zeyda M, Gollinger K, Todoric J, Kiefer FW, Keck M, Aszmann O, et al. Osteopontin is an activator of human adipose tissue macrophages and directly affects adipocyte function. Endocrinology 2011; 152: 2219-27.

29 Katakam AK, Chipitsyna G, Gong Q, Vancha AR, Gabbeta J, Arafat HA. Streptozotocin (STZ) mediates acute upregulation of serum and pancreatic osteopontin (OPN): a novel islet-protective effect of OPN through inhibition of STZ-induced nitric oxide production. J Endocrinol 
2005; 187: 237-47.

30 Kawamura H, Yokote K, Asaumi S, Kobayashi K, Fujimoto M, Maezawa $\mathrm{Y}$, et al. High glucose-induced upregulation of osteopontin is mediated via Rho/Rho kinase pathway in cultured rat aortic smooth muscle cells. Arterioscler Thromb Vasc Biol 2004; 24: 276-81.

31 Tsuchida A, Yamauchi T, Takekawa S, Hada Y, Ito Y, Maki T, et al. Peroxisome proliferator-activated receptor (PPAR)alpha activation increases adiponectin receptors and reduces obesity-related inflammation in adipose tissue: comparison of activation of PPARalpha, PPARgamma, and their combination. Diabetes 2005; 54: 3358-70.

32 Guzmán M, Lo Verme J, Fu J, Oveisi F, Blázquez C, Piomelli D. Oleoylethanolamide stimulates lipolysis by activating the nuclear receptor peroxisome proliferator-activated receptor alpha (PPAR-alpha). J Biol Chem 2004; 279: 27849-54.

$33 \mathrm{Hu}$ Y, Chen Y, Ding L, He X, Takahashi Y, Gao Y, et al. Pathogenic role of diabetes-induced PPAR- $\alpha$ down-regulation in microvascular dysfunction. Proc Natl Acad Sci U S A 2013; 110: 15401-6.

34 Barger PM, Browning AC, Garner AN, Kelly DP. p38 Mitogen-activated protein kinase activates peroxisome proliferator-activated receptor alpha: a potential role in the cardiac metabolic stress response. J Biol Chem 2001; 276: 44495-501.

35 Lu N, Gao Y, Ling Y, Chen Y, Yang Y, Gu HY, et al. Wogonin suppresses tumor growth in vivo and VEGF-induced angiogenesis through inhibiting tyrosine phosphorylation of VEGFR2. Life Sci 2008; 82: 956-63.

36 Carlson CJ, Koterski S, Sciotti RJ, Poccard GB, Rondinone CM. Enhanced basal activation of mitogen-activated protein kinases in adipocytes from type 2 diabetes: potential role of p38 in the downregulation of GLUT4 expression. Diabetes 2003; 52: 634-41.

37 Rajasekar N, Dwivedi S, Nath C, Hanif K, Shukla R. Protection of streptozotocin induced insulin receptor dysfunction, neuroinflam- mation and amyloidogenesis in astrocytes by insulin. Neuropharmacology 2014; 86: 337-52.

38 Taniguchi K, Xia L, Goldberg HJ, Lee KW, Shah A, Stavar L, et al. Inhibition of Src kinase blocks high glucose-induced EGFR transactivation and collagen synthesis in mesangial cells and prevents diabetic nephropathy in mice. Diabetes 2013; 62: 3874-86.

39 Gao P, Wu X, Shui H, Jia R. Fluvastatin inhibits high glucose-induced nuclear factor kappa B activation in renal tubular epithelial cells. J Nephrol 2013; 26: 289-96.

40 Sharma R, Buras E, Terashima T, Serrano F, Massaad CA, Hu L, et al. Hyperglycemia induces oxidative stress and impairs axonal transport rates in mice. PLoS One 2010; 5: e13463.

41 Sodhi CP, Phadke SA, Batlle D, Sahai A. Hypoxia stimulates osteopontin expression and proliferation of cultured vascular smooth muscle cells: potentiation by high glucose. Diabetes 2001; 50 : 1482-90.

42 Bertola A, Deveaux V, Bonnafous S, Rousseau D, Anty R, Wakkach A, et al. Elevated expression of osteopontin may be related to adipose tissue macrophage accumulation and liver steatosis in morbid obesity. Diabetes 2009; 58: 125-33.

43 Gómez-Ambrosi J, Catalán V, Ramírez B, Rodríguez A, Colina I, Silva C, et al. Plasma osteopontin levels and expression in adipose tissue are increased in obesity. J Clin Endocrinol Metab 2007; 92: 3719-27.

44 You JS, Ji HI, Chang KJ, Yoo MC, Yang HI, Jeong IK, et al. Serum osteopontin concentration is decreased by exercise-induced fat loss but is not correlated with body fat percentage in obese humans. Mol Med Rep 2013; 8: 579-84.

45 Ahmad R, Al-Mass A, Al-Ghawas D, Shareif N, Zghoul N, Melhem $\mathrm{M}$, et al. Interaction of osteopontin with IL-18 in obese individuals: implications for insulin resistance. PLoS One 2013; 8: e63944. 